\title{
Correspondence
}

\section{Epidural catheter disconnections}

\section{To the Editor:}

Probably the most common and rarely reported complication of epidural analgesia used for postoperative or labour pain control is the separation of the epidural catheter from its connector at the Huber adapter with resultant contamination of the proximal end of the catheter (Figures A and B). Some institutions have found that catheter disconnection is the most common cause for discontinuation of epidural analgesia. ${ }^{1}$

In spite of recommendations to the contrary ${ }^{2}$ these catheters are often reconnected by nurses, physicians and even on one occasion by the patient's visitors! Sometimes the catheter is cleansed with proviodine or isopropyl alcohol before reconnection, but more often the catheter is simply reattached when it is discovered lying on the bedsheets. Reconnection of the catheter puts the patient at increased risk for the introduction of microbes into the epidural space and the potential for an epidural space infection.

We recommend that, after securing the epidural catheter into the Huber connector, a protective piece of adhesive (or similar product) tape be securely attached as a "flag" around the catheter and the Luer/Huber con-

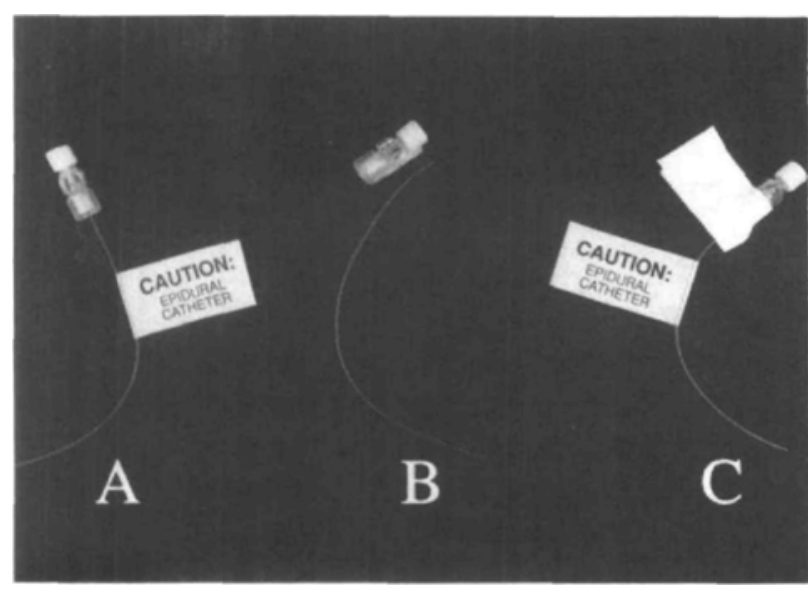

FIGURE (A) Intact Luer/Huber adapter - epidural catheter connection. (B) epidural catheter disconnected from connector at Huber adapter; (c) "flag" sccurely attached to catheter and adapter preventing disconnection. nector (Figure C). This tape is firmly applied to the connector and catheter to eliminate any chance of disconnection and maintain the integrity and sterility of the system. The "flag" can also be used to pin the connector to the front of the patient's gown or clothes to allow ready access to the catheter. One might be tempted to use the warning label enclosed in most epidural kits as a "flag" (Figure A) but we have found that it is not suitable for this purpose as it is too stiff and will not properly adhere to the adapter.

We have begun using this technique for our acute pain service and obstetrical epidurals and we have had no problems with catheter disconnections.

Robin J. Gavelin MD FRCPC

Kenneth W. Patterson FFARCS1

Department of Anesthesiology

The University of Texas Medical Branch

Galveston, TX 77555-0591

\section{REFERENCES}

1 Lubenow T, Ivankovich A. Postoperative epidural analgesia. Critical Care Nursing Clinics of North America $199 \mathrm{I}_{\text {; }}$ 3: 25-34.

2 Litwick $K$, Lubenow $T$. Practical points in the management of continuous epidural infusions. Journal of Post Anesthesia Nursing 1989; 4: 327-30.

\section{General vs regional anaesthesia for minor surgery}

To the Editor;

The popularity of regional anaesthesia (RA) has experienced a steady resurgence during the past decade. Although studies have shown that practicing anaesthetists would prefer to receive RA to general anaesthesia (GA) for both emergency and elective peripheral surgery, ${ }^{1,2} \mathrm{GA}$ remains the technique of choice for most procedures. We conducted this survey of practicing anaesthetists to assess the use of RA in minor surgery and to examine the demographic and educational factors which may influence their choice of anaesthetic technique.

A total of 989 questionnaires was sent to all anaesthetists practicing in Ontario. In addition to demographic and educational data, respondents were asked which anaesthetic technique they would prefer if they were (a) 